\title{
Ion-selective Electrode Array based on a Bayesian Nonlinear Source Separation Method
}

\author{
Leonardo Tomazeli Duarte ${ }^{1 \star}$, Christian Jutten ${ }^{1}$, and Saïd Moussaoui ${ }^{2}$ \\ 1 GIPSA-lab, Institut Polytechnique de Grenoble, CNRS, France \\ Leonardo.duarte@gipsa-lab.inpg.fr, Christian.Jutten@gipsa-lab.inpg.fr \\ 2 IRCCyN, UMR CNRS 6597, Ecole Centrale Nantes, France \\ Said.Moussaoui@irccyn.ec-nantes.fr
}

\begin{abstract}
Ion-selective electrodes (ISE) offer a practical approach for estimating ionic activities. Nonetheless, such devices are not selective, i.e., the ISE response can be affected by interfering ions other than the target one. With the aim of overcoming this problem, we propose a Bayesian nonlinear source separation method for processing the data acquired by an ISE array. The Bayesian framework permits us to easily incorporate prior information such as the non-negativity of the sources into the separation method. The effectiveness of our proposal is attested by experiments using artificial and real data.
\end{abstract}

\section{Introduction}

Ion-selective electrodes (ISEs) are devices used for measuring the ionic activity, a measure of effective concentration of an ion in aqueous solution [1]. In contrast to more sophisticated analytical techniques, an ISE distinguishes itself because of its low cost and its ease of manipulation. Nonetheless, a well-known problem associated with an ISE regards its lack of selectivity [1,2], i.e., the ISE response can be affected by interfering ions other than the target one.

One possible way to deal with the interference problem relies on the use of an electrode array followed by a signal processing block designed for extracting the relevant information from the acquired data. If, for instance, signal processing blocks based on blind source separation (BSS) techniques are considered [3], then one may skip almost totally ${ }^{3}$ the usual calibration stage, which, for an ISE array, is extremely time-demanding and must be performed from time to time due to the electrodes' drift. However, there are several challenging points that make difficult the design of a BSS method for this case. Firstly, the mixing model associated with an ISE array is nonlinear. Secondly, in a real application, the number of samples is usually reduced and the typical assumption of statistical independence between the sources may be not realistic in some scenarios.

* L. T. Duarte is grateful to the CNPq (Brazil) for funding his $\mathrm{PhD}$ research. The authors are grateful to Pierre Temple-Boyer, Jérôme Launay and Ahmed Benyahia (LAAS-CNRS) for the support in the acquisition of the dataset used in this work.

${ }^{3}$ Due to the usual scale indeterminacy of BSS methods, at least one calibration point is necessary. 
In this work, we propose a Bayesian nonlinear BSS algorithm in order to overcome the above-mentioned difficulties. Our motivation is twofold. Firstly, by relying on a Bayesian BSS framework, we can easily exploit prior information other than the statistical independence $[4,5]$. For instance, the sources in our problem are always non-negative. Secondly, elaborated sampling methods [6, 7], like the Gibbs' sampler, allow an efficient implementation of a Bayesian inference scheme even in high-dimensional and complex models as the one treated in this work. Concerning the organization of the paper, we start, in Section 2, with a description of the mixing model associated with an ISE array. In Section 3, we describe the proposed Bayesian BSS method. In Section 4, experiments are carried out. Finally, in Section 5, we state our conclusions.

\section{Mixing model}

Let us consider the analysis of a solution containing $n_{s}$ different kinds of ions via an array of $n_{c}$ ISEs. Due to the interference problem, the response $y_{i t}$ of the $i$-th ISE in the array at the instant $t$ is dependent not only on the activity of its target ion $s_{i t}$ but also on the activities of the other ions in the solution, which are represented by $s_{j t}$. The Nicolsky-Eisenman (NE) equation [1] states that

$$
y_{i t}=e_{i}+d_{i} \log \left(s_{i t}+\sum_{j=1, j \neq i}^{n_{s}} a_{i j} s_{j t}^{z_{i} / z_{j}}\right),
$$

where $e_{i}$ is a constant, $z_{j}$ is the valence of the $j$-th ion and $a_{i j}$ denotes the selectivity coefficients. Finally, $d_{i}=R T / z_{i} F$, where $R$ is the gas constant, $T$ the temperature in Kelvin, and $F$ the Faraday constant. In a matrix representation, the data provided by the ISE array can be described as follows

$$
\mathbf{Y}=\mathbf{e} \cdot \mathbf{1}_{1 \times n_{d}}+\operatorname{diag}(\mathbf{d}) \log \left(\mathbf{A} \otimes^{\mathbf{z}} \mathbf{S}\right),
$$

where $n_{d}$ denotes the number of samples, $\mathbf{Y} \in \mathbb{R}^{n_{c} \times n_{d}}, \mathbf{e}=\left[e_{1}, \ldots, e_{n_{c}}\right]^{T}, \mathbf{d}=$ $\left[d_{1}, \ldots, d_{n_{c}}\right]^{T}$. Matrix $\mathbf{A} \in \mathbb{R}_{+}^{n_{c} \times n_{s}}$ contains the selectivity coefficients $a_{i j}$. The $j$-th row of the matrix $\mathbf{S} \in \mathbb{R}_{+}^{n_{s} \times n_{d}}$ corresponds to the temporal evolution of the activity of the $j$-th ion. Finally, $\mathbf{z}=\left[z_{1}, \ldots, z_{n_{s}}\right]^{T}$, and the operator $\otimes^{\mathbf{z}}$ describes the nonlinear transformation inside the log term in the NE model (see (1)). If the valences $z_{i}$ are equal, then $\otimes^{\mathbf{z}}$ results in a simple matrix multiplication and, thus, (2) becomes a particular case of the class of post-nonlinear (PNL) models [8].

In view of a possible model inaccuracy and/or of the errors introduced by the measurement system, a more realistic description of the ISE array is given by $\mathbf{X}=\mathbf{Y}+\mathbf{N}$, where $\mathbf{N} \in \mathbb{R}^{n_{c} \times n_{d}}$ represents the noise terms. We assume a zero mean white Gaussian noise with covariance matrix $\mathbf{C}=\operatorname{diag}\left(\left[\sigma_{1}^{2}, \ldots, \sigma_{n_{c}}^{2}\right]\right)$. Finally, $S N R_{i}$ denotes the resulting signal-to-noise ratio at the ISE $i$.

\section{Bayesian Source Separation Method}

Given the mixing model description, we can now formulate the source separation problem treated in this work: estimate the elements of $\mathbf{S}$ by using the ISE array 
response $\mathbf{X}$ and by assuming that the vector of valences $\mathbf{z}$ is known. Since we envisage a blind method, all the parameters related to the mixing model (except z) and the noise variance at each electrode are unknown and, thus, should be estimated. Furthermore, as it will become clear later, there are other unknown parameters, denoted by $\phi$, which are related to the prior distributions assigned to the sources. Henceforth, all these parameters will be represented by the vector $\boldsymbol{\theta}=[\mathbf{S}, \mathbf{A}, \mathbf{d}, \mathbf{e}, \boldsymbol{\sigma}, \boldsymbol{\phi}]$ and we will adopt the following notation: $\boldsymbol{\theta}_{-\theta_{q}}$ represents the vector containing all elements of $\boldsymbol{\theta}$ except $\theta_{q}$.

A first step in the development of a Bayesian method concerns the definition of prior distributions for each element of $\boldsymbol{\theta}$. Then, by relying on the model likelihood and on the Bayes' rule, one can obtain the posterior distribution of $\boldsymbol{\theta}$ given X. Finally, we use Markov Chain Monte Carlo methods to generate samples from the posterior distribution. This permits us to approximate the Bayesian minimum mean square error (MMSE) estimator [9] for $\boldsymbol{\theta}$ in a straightforward manner. In the sequel, we shall detail each of these steps.

\subsection{Priors definition}

Ionic activities: The sources are assumed i.i.d. and mutually independent. Also, since the sources represent ionic activities, it is natural to consider a non-negative prior distribution. In this context, [4] has shown that a modeling based on Gamma distributions provides a flexible solution, since it can model from sparse to almost uniform sources. However, in this work, we adopt a $\operatorname{lognormal}$ distribution ${ }^{4}$ for each source, i.e., $p\left(s_{j t}\right)=\log \mathcal{N}\left(\mu_{s_{j}}, \sigma_{s_{j}}^{2}\right)$ since one can find a conjugate prior ${ }^{5}$ for the estimation of the unknown parameters $\phi_{j}=\left[\mu_{s_{j}} \sigma_{s_{j}}^{2}\right]$. Indeed, this can be done by setting the priors $p\left(\mu_{s_{j}}\right)=\mathcal{N}\left(\tilde{\mu}_{s_{j}}, \tilde{\sigma}_{s_{j}}^{2}\right)$ and $p\left(1 / \sigma_{s_{j}}^{2}\right)=\mathcal{G}\left(\alpha_{\sigma_{s_{j}}}, \beta_{\sigma_{s_{j}}}\right)$, where $\tilde{\mu}_{s_{j}}, \tilde{\sigma}_{s_{j}}, \alpha_{\sigma_{s_{j}}}, \beta_{\sigma_{s_{j}}}$ are hyperparameters. Also, there is a practical argument behind the choice of a log-normal distribution. Ionic activities are expected to have a small variation in the logarithmic scale. This particularity can be taken into account by the log-normal distribution, since such a distribution is nothing but a Gaussian distribution in the logarithmic scale.

Selectivity coefficients: The parameters $a_{i j}$ are also non-negative $[1,2]$. Moreover, it is rare to find a sensor whose response depends more on the interfering ion than on the target one. Given that, we assume that $a_{i j}$ is uniformly distributed in $[0,1]$, i.e., $p\left(a_{i j}\right)=\mathcal{U}(0,1)$.

Non-linear model parameters: Since the parameters $d_{i}$ are related to physical parameters, a priori, they could be known beforehand. For instance, at room temperature, one has $d_{i}=0.059 / z_{i}$, and the ISEs with such sensibility are said to have a Nernstian response. However, in a practical scenario, due to the sensor

\footnotetext{
${ }^{4}$ The following notation is used. $\mathcal{N}\left(\mu, \sigma^{2}\right), \log \mathcal{N}\left(\mu, \sigma^{2}\right), \mathcal{G}(\alpha, \beta)$ and $\mathcal{U}(a, b)$ represent the Gaussian, Lognormal, Gamma and Uniform distributions, respectively.

${ }^{5}$ From the Bayes' rule $p(X / Y) \propto p(Y / X) p(X)$. Then $p(X)$ is a conjugate prior with respect to the likelihood $p(Y / X)$ when $p(X / Y)$ and $p(X)$ belong to the same family. Conjugate priors ease the simulations conducted in a MCMC algorithm.
} 
fabrication process and to aging, an important deviation from this theoretical value can be observed. In order to take into account this deviation, we apply a Gaussian prior $p\left(d_{i}\right)=\mathcal{N}\left(\mu_{d_{i}}=0.059 / z_{i}, \sigma_{d_{i}}^{2}\right)$, where $\sigma_{d_{i}}$ is an hyperparameter. We assume that the elements of the vector $\mathbf{d}$ are statistically independent.

Offset parameters: In contrast to the parameters $d_{i}$, there is no theoretical value for $e_{i}$. However, this parameter usually [10] lies on the interval [.05, 0.35]. Hence, we can set $p\left(e_{i}\right)=\mathcal{N}\left(\mu_{e_{i}}=0.20, \sigma_{e_{i}}^{2}\right)$ where the variance $\sigma_{e_{i}}^{2}$ must be defined so that the resulting prior goes toward a non-informative prior in this interval (for instance, see [6] for a discussion on non-informative priors.). The elements of the vector e are assumed mutually independent.

Noise variances: Finally, we assign inverse Gamma distributions for the noise variances, i.e., $p\left(1 / \sigma_{i}^{2}\right)=\mathcal{G}\left(\alpha_{\sigma_{i}}, \beta_{\sigma_{i}}\right)$. By proceeding this way, one obtains a conjugate pair, which eases the sampling step. Moreover, it is possible to set the hyperparameters $\alpha_{\sigma_{i}}$ and $\beta_{\sigma_{i}}$ to obtain a non-informative prior [4].

\subsection{The posterior distribution}

A first step to obtain the posterior of $\boldsymbol{\theta}$ is to find the likelihood $p(\mathbf{X} \mid \boldsymbol{\theta})$ associated with the mixing model. From the assumption of i.i.d. Gaussian noise which is also spatially uncorrelated, it asserts that

$$
p(\mathbf{X} \mid \boldsymbol{\theta})=\prod_{t=1}^{n_{d}} \prod_{i=1}^{n_{c}} \mathcal{N}_{x_{i t}}\left(e_{i}+d_{i} \log \left(\sum_{j=1}^{n_{s}} a_{i j} s_{j t}^{z_{i} / z_{j}}\right), \sigma_{i}^{2}\right),
$$

where $\mathcal{N}_{x_{i k}}\left(\mu, \sigma^{2}\right)$ is a Gaussian distribution in $x_{i k}$ with parameters $\mu$ and $\sigma^{2}$.

Having defined the likelihood $p(\mathbf{X} \mid \boldsymbol{\theta})$ and the prior distribution $p(\boldsymbol{\theta})$, we can use the Bayes' rule to write the posterior distribution as $p(\boldsymbol{\theta} \mid \mathbf{X}) \propto p(\mathbf{X} \mid \boldsymbol{\theta}) p(\boldsymbol{\theta})$. Since the unknown variables of our problem are, by assumption, mutually independent (except $\mathbf{S}$ and $\boldsymbol{\phi}$ ), we can factorize $p(\boldsymbol{\theta} \mid \mathbf{X})$ in the following manner

$$
p(\boldsymbol{\theta} \mid \mathbf{X}) \propto p(\mathbf{X} \mid \boldsymbol{\theta}) \cdot p(\mathbf{S} \mid \boldsymbol{\phi}) \cdot p(\boldsymbol{\phi}) \cdot p(\mathbf{A}) \cdot p(\mathbf{e}) \cdot p(\mathbf{d}) \cdot p(\boldsymbol{\sigma}) .
$$

With the posterior distribution in hand we can set an inference scheme. In this work, we consider the Bayesian minimum mean square error (MMSE) estimator [9] which is defined as $\boldsymbol{\theta}_{M M S E}=\int \boldsymbol{\theta} p(\boldsymbol{\theta} \mid \mathbf{X}) d \boldsymbol{\theta}$. The problem here is the analytical resolution of this integral, which culminates in a complex task when one deals with a high-dimensional problem with non-standard distributions [6].

A possible approach to overcome the calculation of the integral related to $\boldsymbol{\theta}_{M M S E}$ is to generate samples from the posterior distribution $p(\boldsymbol{\theta} \mid \mathbf{X})$ and then approximate the MMSE estimator using these samples. If, for instance, the generated samples are represented by $\boldsymbol{\theta}^{1}, \boldsymbol{\theta}^{2}, \ldots, \boldsymbol{\theta}^{M}$, then the MMSE estimator can be approximated by $\widetilde{\boldsymbol{\theta}}_{M M S E}=\frac{1}{M} \sum_{i=1}^{M} \boldsymbol{\theta}^{i}$. According to the law of large numbers, $\widetilde{\boldsymbol{\theta}}_{M M S E}=\boldsymbol{\theta}_{M M S E}$ as $M \rightarrow+\infty$. This important result gives the theoretical foundation for the above described methodology, which is referred as Monte Carlo integration [6]. In the next section, we apply this methodology to the estimation problem treated in this work. 


\subsection{Bayesian inference through the Gibbs sampler}

The generation of samples from $p(\boldsymbol{\theta} \mid \mathbf{X})$ is performed by the Gibbs sampler. Given each conditional distribution $p\left(\theta_{i} \mid \boldsymbol{\theta}_{-\theta_{i}}, \mathbf{X}\right)$, the Gibbs sampler uses the procedure described in Tab. 1 to generate a Markov Chain having $p(\boldsymbol{\theta} \mid \mathbf{X})$ as stationary distribution. Therefore, after a burn-in period, which is necessary for the generated Markov Chain to reach its stationary distribution, the algorithm given Tab. 1 provides samples from $p(\boldsymbol{\theta} \mid \mathbf{X})$. Then, as discussed before, one can use these samples to obtain the MMSE estimator of $\boldsymbol{\theta}$.

Table 1. Simulation of $p(\boldsymbol{\theta} \mid \mathbf{X})$ through the Gibbs' sampler

1. Initialize the actual samples $\boldsymbol{\theta}^{0}=\left[\theta_{1}^{0}, \theta_{2}^{0}, \ldots, \theta_{N}^{0}\right]$;

2. For $p=1$ to $P$ do

$$
\begin{aligned}
\theta_{1}^{p} & \sim p\left(\theta_{1} \mid \boldsymbol{\theta}_{-\theta_{1}}^{p}, \mathbf{X}\right) \\
\theta_{2}^{p} & \sim p\left(\theta_{2} \mid \boldsymbol{\theta}_{-\theta_{2}}^{p}, \mathbf{X}\right) \\
\vdots & \\
\theta_{N}^{p} & \sim p\left(\theta_{N} \mid \boldsymbol{\theta}_{-\theta_{N}}^{p}, \mathbf{X}\right)
\end{aligned}
$$

end

The conditional densities $p\left(\theta_{q} \mid \boldsymbol{\theta}_{-\theta_{q}}^{p}, \mathbf{X}\right)$ are summarized in Tab 2. Due to the reduced space, the derivation of these expressions is omitted here. It can be noted that, because of the selected priors, we obtained conjugate pairs for almost all parameters. As a consequence, the sampling in these cases becomes straightforward. However, for $p\left(s_{j t} \mid \boldsymbol{\theta}_{-s_{j t}}, \mathbf{X}\right)$ and $p\left(a_{i j} \mid \boldsymbol{\theta}_{-a_{i j}}, \mathbf{X}\right)$, we obtained the following non-standard distributions:

$$
\begin{aligned}
& p\left(s_{j t} \mid \boldsymbol{\theta}_{-s_{j t}}, \mathbf{X}\right) \propto \exp {\left[\sum_{i=1}^{n_{c}}-\frac{1}{2 \sigma_{i}^{2}}\left(x_{i t}-e_{i}-\right.\right.} \\
&\left.\left.d_{i} \log \left(a_{i j} s_{j t}^{z_{j} / z_{j}}+\sum_{\ell=1, \ell \neq j}^{n_{s}} a_{i \ell} s_{\ell t}^{z_{i} / z_{\ell}}\right)\right)^{2}-\frac{\left(\log \left(s_{j t}\right)-\mu_{j}\right)^{2}}{2 \sigma_{j}^{2}}\right] \frac{1}{s_{j t}} \mathbb{1}_{[0,+\infty[}, \\
& p\left(a_{i j} \mid \boldsymbol{\theta}_{-a_{i j}}, \mathbf{X}\right) \propto \exp \left[-\frac{1}{2 \sigma_{i}^{2}} \sum_{t=1}^{n_{t}}\left(x_{i t}-e_{i}-\right.\right. \\
&\left.\left.d_{i} \log \left(a_{i j} s_{j t}^{z_{i} / z_{j}}+\sum_{\ell=1, \ell \neq j}^{n_{s}} a_{i \ell} s_{\ell t}^{z_{i} / z_{\ell}}\right)\right)^{2}\right] \mathbb{1}_{[0,1]},
\end{aligned}
$$

where $\mathbb{1}$ denotes the indicator function. The sampling from these distributions is conducted through the Metropolis-Hasting ( $\mathrm{MH}$ ) algorithm [6]. A truncated Gaussian distribution was adopted as instrumental distribution. 
Table 2. Prior and conditional distributions of the Bayesian model parameters.

\begin{tabular}{|c|c|c|c|}
\hline$\overline{\theta_{q}}$ & $p\left(\theta_{q} \mid \boldsymbol{\theta}_{-\theta_{q}}, \mathbf{X}\right)$ & Auxiliary parameters & Prior $p\left(\theta_{q}\right)$ \\
\hline$\overline{s_{j k}}$ & see Eq. (5) & - & $\log \mathcal{N}\left(\mu_{s_{j}}, \sigma_{s_{j}}^{2}\right)$ \\
\hline$a_{i j}$ & see Eq. (6) & - & $\mathcal{U}(0,1)$ \\
\hline$\mu_{s_{j}}$ & $\begin{array}{c}\mathcal{N}\left(\frac{\mu_{L_{j}} \tilde{\sigma}_{s_{j}}^{2}+\tilde{\mu}_{s_{j}} \sigma_{L_{j}}^{2}}{\sigma_{L_{j}}^{2}+\tilde{\sigma}_{s_{j}}^{2}},\right. \\
\left.\frac{\sigma_{L_{j}}^{2} \tilde{\sigma}_{s_{j}}^{2}}{\sigma_{L_{j}}^{2}+\tilde{\sigma}_{s_{j}}^{2}}\right)\end{array}$ & $\begin{array}{c}\mu_{L_{j}}=\frac{\sum_{t=1}^{n_{d}} \log \left(s_{j t}\right)}{n_{d}} \\
\sigma_{L_{j}}^{2}=\frac{\tilde{\sigma}_{s_{j}}^{2}}{n_{d}}\end{array}$ & $\mathcal{N}\left(\tilde{\mu}_{s_{j}}, \tilde{\sigma}_{s_{j}}^{2}\right)$ \\
\hline $1 / \sigma_{s_{j}}^{2}$ & $\mathcal{G}\left(\alpha_{P_{j}}, \beta_{P_{j}}\right)$ & $\begin{array}{c}\alpha_{P_{j}}=\alpha_{\sigma_{s_{j}}}+n_{d} / 2 \\
\beta_{P_{j}}^{-1}=0.5 \sum_{t=1}^{n_{d}}\left(\log \left(s_{j t}\right)-\mu_{s_{j}}\right)^{2}-\beta_{\sigma_{s_{j}}}^{-1}\end{array}$ & $\mathcal{G}\left(\alpha_{\sigma_{s_{j}}}, \beta_{\sigma_{s_{j}}}\right)$ \\
\hline$d_{i}$ & $\begin{array}{c}\mathcal{N}\left(\frac{\mu_{L_{d_{i}}} \sigma_{d_{i}}^{2}+\mu_{d_{i}} \sigma_{L_{d_{i}}}^{2}}{\sigma_{L_{d_{i}}}^{2}+\sigma_{d_{i}}^{2}}\right. \\
\left.\frac{\sigma_{L_{d_{i}}}^{2} \sigma_{d_{i}}^{2}}{\sigma_{L_{d_{i}}}^{2}+\sigma_{d_{i}}^{2}}\right)\end{array}$ & $\begin{aligned} \mu_{L_{d_{i}}} & =\frac{\left(\sum_{t=1}^{n_{d}}\left(x_{i t}-e_{i}\right)\right) \log \left(\sum_{\ell=1}^{n_{s} a_{i \ell} s_{\ell \ell}^{\frac{z_{i}}{z_{\ell}}}}\right)}{\left(\log \left(\sum_{\ell=1}^{n_{s} a_{i \ell} s_{\ell t}} \frac{z_{i}}{z_{\ell \ell}}\right)\right)^{2}} \\
\sigma_{L_{d_{i}}}^{2} & =\sigma_{d_{i}}^{2}\left(\log \left(\sum_{\ell=1}^{n_{s}} a_{i \ell} s_{\ell t}^{\frac{z_{i}}{z_{\ell}}}\right)\right)^{-2}\end{aligned}$ & $\mathcal{N}\left(\mu_{d_{i}}, \sigma_{d_{i}}^{2}\right)$ \\
\hline$e_{i}$ & 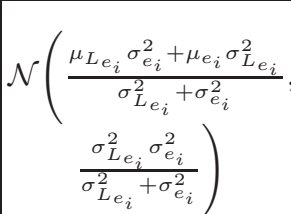 & $\begin{array}{c}\mu_{L_{e_{i}}}=\frac{\sum_{t=1}^{n_{d}}\left(x_{i t}-d_{i} \log \left(\sum_{\ell=1}^{n_{s}} a_{i \ell} \frac{\frac{z_{i}}{z_{\ell \ell}}}{\ell_{d}}\right)\right.}{n_{d}} \\
\sigma_{L_{e_{i}}}^{2}=\sigma_{e_{i}}^{2} n_{d}^{-1}\end{array}$ & $\mathcal{N}\left(\mu_{e_{i}}, \sigma_{e_{i}}^{2}\right)$ \\
\hline $1 / \sigma_{i}^{2}$ & $\mathcal{G}\left(\alpha_{P_{i}}, \beta_{P_{i}}\right)$ & $\begin{array}{c}\alpha_{P_{i}}=\alpha_{\sigma_{i}}+\frac{n_{d}}{2}, \beta_{P_{i}}^{-1}=\Psi-\beta_{\sigma_{i}}^{-1} \\
\Psi=0.5 \sum_{t=1}^{n_{d}}\left(x_{i t}-e_{i}-d_{i} \log \left(\sum_{\ell=1}^{n_{s} a_{i \ell}} \begin{array}{c}\frac{z_{i}}{z_{\ell t}} \\
\ell\end{array}\right)\right)^{2}\end{array}$ & $\mathcal{G}\left(\alpha_{\sigma_{i}}, \beta_{\sigma_{i}}\right)$ \\
\hline
\end{tabular}

\section{Experimental Results}

We test our algorithm using artificial data and also in a real situation involving an array constituted of electrodes of potassium $\left(\mathrm{K}^{+}\right)$and of ammonium $\left(\mathrm{NH}_{4}^{+}\right)$. The quality related to the estimation $\widehat{s}_{i}(t)$ (after scale normalization) of the source $s_{i}(t)$ is assessed using the following index: $S I R_{i}=10 \log \left(\frac{E\left\{s_{i}(t)^{2}\right\}}{E\left\{\left(s_{i}(t)-\widehat{s}_{i}(t)\right)^{2}\right\}}\right)$. Experiments with artificial data: Aiming to define testing scenarios that are as close as possible to real situations, we make use of the selectivity coefficients database presented in [2]. Concerning the sources, we considered a set of $n_{d}=900$ samples endowed with a temporal structure, each sample being generated by a lognormal distribution. In a first situation, we simulate an array of two electrodes $\left(n_{c}=2\right)$ (each one having a different ion as target) for estimating the activities of $\mathrm{NH}_{4}^{+}$and $\mathrm{K}^{+}\left(n_{s}=2\right)$. As discussed in Section 2, one has a PNL model in this situation given that the valences are equal $\left(z_{1}=z_{2}=1\right)$. The parameters of the mixing system for this case were $a_{12}=0.3, a_{21}=0.4, d_{1}=0.056, d_{2}=0.056$, $e_{1}=0.090, e_{2}=0.105$ and $S N R_{1}=S N R_{2}=18 \mathrm{~dB}$. Concerning the Gibbs sampler, 3000 iterations with a burn-in period of 1400 were conducted. The 
burn-in value was fixed empirically after a visual inspection of the chains and no convergence monitoring strategy was applied. Our method was able to provide a good estimate of the sources in this situation. The performance indexes for this case were $S I R_{1}=19.84 \mathrm{~dB}, S I R_{2}=18.75 \mathrm{~dB}$ and $S I R=19.29 \mathrm{~dB}$.

We also analyze the more complicate case of estimating the activities of calcium $\left(\mathrm{Ca}^{2+}\right)$ and sodium $\left(\mathrm{Na}^{+}\right)$using an array of two ISEs $\left(n_{c}=2\right.$ and $n_{s}=$ 2 ), each one having a different ion as target. Since the valences are different now, the mixing system is composed of a nonlinear mapping followed by componentwise logarithm functions (see Eq. (1)). We consider the same source waveforms of the last case and the mixing system parameters were $a_{12}=0.3, a_{21}=0.4$, $d_{1}=0.026, d_{2}=0.046, e_{1}=0.090, e_{2}=0.105$ and $S N R_{1}=S N R_{2}=18 \mathrm{~dB}$. The performance indexes in this case were $S I R_{1}=14.96 \mathrm{~dB}, S I R_{2}=17.37 \mathrm{~dB}$ and $S I R=16.17 \mathrm{~dB}$. Despite the performance deterioration with respect to the first case, our method provided fair estimations for the sources. The number of iterations for the Gibbs sampler was 5000 with a burn-in period of 2000 .

Experiments with real data: We consider the analysis of a solution containing $\mathrm{K}^{+}$and $\mathrm{NH}_{4}^{+}$through an array composed of one $\mathrm{K}^{+}$-ISE and one $\mathrm{NH}_{4}^{+}$-ISE. This situation is typical in water quality monitoring. The actual sources and the data acquired $\left(n_{d}=169\right)$ by the array are shown in the left and in the right side, respectively, of Fig. 1. For the Gibbs' sampler, we considered 3000 iterations and a burn-in period of 1000. In Fig. 1, we plot the retrieved sources. The performance indexes in this case were $S I R_{1}=25.10 \mathrm{~dB}, S I R_{2}=23.69 \mathrm{~dB}, S I R=24.39 \mathrm{~dB}$. Despite a small residual interference, mainly for the $K^{+}$activity, our method was able to provide a good estimation even in this difficult scenario in which the sources are dependent and only a reduced number of samples is available. Conversely, this situation poses a problem to ICA-based methods. Indeed, the performance of the PNL-ICA method proposed in [11] was poor in this case $\left(S I R_{1}=7.67 \mathrm{~dB}, S I R_{2}=-0.33 \mathrm{~dB}, S I R=3.67 \mathrm{~dB}\right)$.
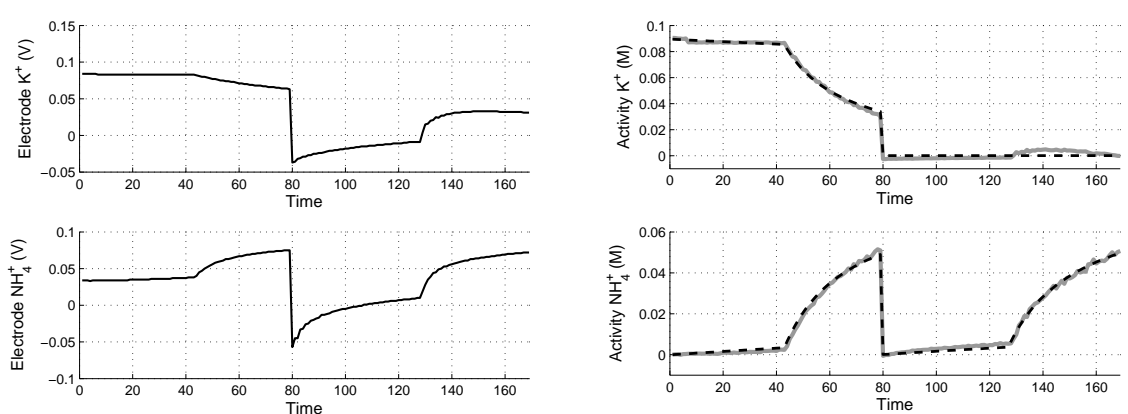

Fig. 1. Left: mixtures. Right: actual sources (dashed black) and estimations (after scale normalization) provided by the Bayesian method (gray). 


\section{Conclusion}

In this work, we developed a MCMC-based Bayesian nonlinear BSS method for processing the outputs of an array of ion-selective electrodes. The Bayesian framework allowed us to consider prior information such as the non-negativity of the sources and of the selectivity coefficients. Experiments with artificial and real data attested the viability of our proposal. Moreover, given that in the Bayesian formulation the independence hypothesis is not as important as in ICA methods, our method could provide good estimations even in a scenario where the sources were dependent and where only a reduced number of samples was available.

A first perspective of this work is related to the sources modeling. Indeed, we assume i.i.d. sources which means that we are not taking advantage of the fact that chemical sources do have a time-structure (they are usually slowly varying signals). Also, there are other two points that deserve further investigation: 1) the case where neither the number of ions in the solution nor their valences are available. 2) Although widely used, the NE equation may be not precise in some cases [12]. Therefore, by considering more precise models, one could obtain a better separation and then work even when a very high precision is needed.

\section{References}

1. Fabry, P., Fouletier, J., eds.: Microcapteurs chimiques et biologiques. Lavoisier (2003) In French.

2. Umezawa, Y., Bühlmann, P., Umezawa, K., Tohda, K., Amemiya, S.: Potentiometric selectivity coefficients of ion-selective electrodes. Pure and Applied Chemistry 72 (2000) 1851-2082

3. Duarte, L.T., Jutten, C.: Blind source separation of a class of nonlinear mixtures. LNCS (Proc. of ICA 2007) 4666 (2007) 41-48

4. Moussaoui, S., Brie, D., Mohammad-Djafari, A., Carteret, C.: Separation of nonnegative mixture of non-negative sources using a bayesian approach and mcmc sampling. IEEE Trans. on Signal Processing 54(11) (2006) 4133-4145

5. Févotte, C., Godsill, S.J.: A Bayesian approach for blind separation of sparse sources. IEEE Trans. on Audio, Speech and Language Processing 14 (2006) 2174 2188

6. Robert, C.P.: The Bayesian Choice. Springer (2007)

7. Gilks, W.R., Richardson, S., Spiegelhalter, D., eds.: Markov chain Monte Carlo in practice. CRC Press (1995)

8. Taleb, A., Jutten, C.: Source separation in post-nonlinear mixtures. IEEE Trans. on Signal Processing 47(10) (1999) 2807-2820

9. Kay, S.M.: Fundamentals of statistical signal processing: estimation theory. Prentice-Hall (1993)

10. Gründler, P.: Chemical sensors: an introduction for scientists and engineers. Springer (2007)

11. Duarte, L.T., Suyama, R., Attux, R.R.F., Zuben, F.J.V., Romano, J.M.T.: Blind source separation of post-nonlinear mixtures using evolutionary computation and order statistics. LNCS (Proc. of ICA 2006) 3889 (2006) 66-73

12. Bakker, E., Pretsch, E., Bühlmann, P.: Selectivity of poentiometric ion sensors. Analytical Chemistry 72 (2000) 1127-1133 